Proyecciones Journal of Mathematics

Vol. 40, No 5, pp. 1117-1135, October 2021.

doil 10.22199 / issn.0717-6279-4271

Universidad Católica del Norte

Antofagasta - Chile

\title{
Existence of solutions of boundary value problems for fractional differential equations with integral conditions
}

\author{
Ahcene Boukehila \\ University Amar Telidji of Laghouat, Algeria \\ Received : June 2020. Accepted : February 2021
}

\begin{abstract}
In this work we investigate the existence and uniqueness of solutions of boundary value problems for fractional differential equations involving the Caputo fractional derivative with integral conditions and the nonlinear term depends on the fractional derivative of an unknown function. Our existence results are based on Banach contraction principle and Schauder fixed point theorem. Two examples are provided to illustrate our results.
\end{abstract}

Keywords: Fractional differential equation; Green's function; $B a-$ nach contraction principle; Schauder fixed point theorem.

MSC (2010): 26A33; 34B15; 34B27. 


\section{Introduction}

Fractional differential equations are generalizations of the ordinary differential equations to an arbitrary non-integer order. They have attracted considerable interest due to their ability to model complex phenomena in various science and engineering fields $[2,7,12]$. In fact, the fractional differential equations are considered as alternative models to nonlinear differential equations which induced extensive researches in various fields including the theoretical part $[5,9,10,11,13,16]$. Some existence results for boundary value problems with integral conditions can be found in $[1,8,15]$.

In [3], Benchohra et al. established sufficient conditions for the existence of solutions to a class of boundary value problem for fractional differential equations using the techniques of some fixed point theorems.

By means of Banach contraction principle and Leray-Schauder nonlinear alternative, Guezane-Lakoud et al.[6] proved the existence and uniqueness of solutions for boundary value problems for a class of fractional differential equations with fractional integral condition.

However, it is an essential condition that the nonlinear term depends on the unknown function indicated in [3]. Thus, it is desirable to extend this condition which leading to a more difficult and complicated case, in which the aim of the present paper is to overcome these difficulties. Inspired by these works in $[3,6]$ and the references therein, the present study was aimed to investigate the existence and uniqueness of solutions of boundary value problems for the fractional differential equations with integral conditions and the nonlinear term depends on the fractional derivative of an unknown function. Hence, we consider the following fractional boundary value problem of the form

$$
\begin{gathered}
{ }^{c} D_{0^{+}}^{q} u(t)=f\left(t, u(t),{ }^{c} D_{0^{+}}^{\sigma} u(t)\right), \quad 0<t<1, \\
u(0)-u^{\prime}(0)=\int_{0}^{1} \eta_{1}(s) u(s) d s, \\
u(1)-u^{\prime}(1)=\int_{0}^{1} \eta_{2}(s) u(s) d s,
\end{gathered}
$$

where ${ }^{c} D_{0^{+}}^{q}$ is the Caputo fractional derivative of order $q, 1<q \leq 2$ and $0<\sigma<1, f:[0,1] \times \mathbf{R} \times \mathbf{R} \rightarrow \mathbf{R}$ and $\eta_{1}, \eta_{2}$ are the continuous functions which will be specified later. In this paper, we firstly derive the corresponding Green's function. Consequently, problem (1.1)-(1.3) is reduced to equivalent Fredholm integral equation of second kind. Finally, 
using Banach contraction principle and Schauder fixed point theorem the existence and uniqueness of solutions are obtained. The other parts of the paper are organized as follows: In Section 2, we list some definitions and lemmas to be used later. In section 3 , we present and prove our main results which consist of the uniqueness and existence theorems. At the end of this section, two examples are also provided to illustrate the main results.

\section{Preliminaries}

In this section, we give some basic definitions and lamas of fractional calculus $[9,16]$ which will be used in this paper.

Let us denote by $L^{1}([0,1], \mathbf{R})$ the Banach space of Lebesgue integrable functions $f:[0,1] \rightarrow \mathbf{R}$ with the norm

$$
\|y\|_{L^{1}}=\int_{0}^{1}|y(t)| d t .
$$

Definition 2.1. [16] A function $\varphi(x)$ is called absolutely continuous on an interval $\Omega$, if for any $\varepsilon>0$ there exists a $\delta$ such that for any finite set of pairwise nonintersecting intervals $\left[a_{k}, b_{k}\right] \subset \Omega, k=1,2, \ldots, n$, such that $\sum_{k=1}^{n}\left(b_{k}-a_{k}\right)<\delta$ the inequality $\sum_{k=1}^{n}\left|f\left(\left(b_{k}\right)-f\left(a_{k}\right)\right)\right|<\varepsilon$ holds. The space of these functions is denoted by $A C(\Omega)$.

Definition 2.2. [16] For $n \in \mathbf{N}=\{1,2, \ldots\}$, we denote by $A C^{n}(\Omega)$ the space of functions $\varphi(x)$ which have continuous derivatives up to order $n-1$ on $\Omega$ with $\varphi^{(n-1)}(x) \in A C(\Omega)$.

In particular, $A C^{1}(\Omega)=A C(\Omega)$.

Definition 2.3. [9] The Riemann-Liouville fractional integral of order $\alpha>$ 0 for a continuous function $\varphi \in C[a, b]$ is defined as

$$
I_{a^{+}}^{\alpha} \varphi(t)=\frac{1}{\Gamma(\alpha)} \int_{a}^{t} \varphi(s)(t-s)^{(\alpha-1)} d s,
$$

where $\Gamma$ is the gamma function.

Definition 2.4. [9] For a function $\varphi \in A C^{n}[a, b]$, the Riemann-Liouville fractional derivative of order $\alpha \geq 0$ of $\varphi$ is defined as

$$
D_{a^{+}}^{\alpha} \varphi(t)=\frac{1}{\Gamma(n-\alpha)}\left(\frac{d}{d t}\right)^{n} \int_{a}^{t} \varphi(s)(t-s)^{(n-\alpha-1)} d s,
$$

exists almost everywhere on $[a, b]$ where $n=[\alpha]+1$ and $[\alpha]$ is the integer part of $\alpha$. 
Definition 2.5. [9] For a function $\varphi \in A C^{n}[a, b]$, the Caputo fractional derivative of order $\alpha \geq 0$ of $\varphi$ is defined as

$$
{ }^{c} D_{a^{+}}^{\alpha} \varphi(t)=\frac{1}{\Gamma(n-\alpha)} \int_{a}^{t} \varphi^{(n)}(s)(t-s)^{(n-\alpha-1)} d s,
$$

exists almost everywhere on $[a, b]$ where $n=[\alpha]+1$ and $[\alpha]$ is the integer part of $\alpha$.

Lemma 2.6. [9] Let $\alpha>0, \beta>0, f \in L^{1}[0,1]$. Then the operator ${ }^{\alpha}$ has the semigroup property

$$
\begin{aligned}
I_{0^{+}}^{\alpha} I_{0^{+}}^{\beta} f(t) & =I_{0^{+}}^{\alpha+\beta} f(t), \\
& =I_{0^{+}}^{\beta} I_{0^{+}}^{\alpha} f(t),
\end{aligned}
$$

for all $t \in[0,1]$.

Lemma 2.7. (Lemma 10, [6]) Let $\beta>0$ and $f \in L^{1}[0,1]$. Then

$$
I_{0^{+}}^{\beta+1} f(t) \leq\left\|I_{0^{+}}^{\beta} f\right\|_{L^{1}},
$$

for allt $\in[0,1]$.

Lemma 2.8. [9] Let $\beta>\alpha$ and $f \in L^{1}[0,1]$. Then

$$
{ }^{c} D_{0^{+}}^{\alpha} I_{0^{+}}^{\beta} f(t)=I_{0^{+}}^{\beta-\alpha} f(t),
$$

and

$$
{ }^{c} D_{0^{+}}^{\alpha} I^{\alpha} f(t)=f(t),
$$

for allt $\in[0,1]$.

Lemma 2.9. [9] Let $\alpha>0, n-1 \leq \alpha<n, \beta \in\{0, \ldots, n-1\}$ and $f(t)=(t-a)^{\beta}$, then

$$
{ }^{c} D_{a^{+}}^{\alpha} f(t)=0 .
$$

Moreover, if $\beta>n-1$, then

$$
{ }^{c} D_{a^{+}}^{\alpha} f(t)=\frac{\Gamma(\beta+1)}{\Gamma(\beta-\alpha+1)}(t-a)^{\beta-\alpha} .
$$

In particular

$$
{ }^{c} D_{0^{+}}^{\alpha} t^{\beta-1}=\frac{\Gamma(\beta)}{\Gamma(\beta-\alpha)} t^{\beta-\alpha-1}, \quad \beta>n,
$$


Lemma 2.10. [9] The solution to the fractional differential equation ${ }^{c} D_{a^{+}}^{\alpha} \varphi(t)=0$, with $\alpha>0$ and $\varphi \in A C^{n}[0,1]$ has the following form:

$$
\varphi(t)=c_{1}+c_{2} t+c_{3} t^{2}+\ldots+c_{n} t^{n-1},
$$

where $c_{i} \in \mathbf{R}, i=0, \ldots, n$ and $n=[\alpha]+1$.

Lemma 2.11. [9] Let $\alpha>0$ and $\varphi \in A C^{n}[0,1]$. Then

$$
I^{\alpha c} D_{a^{+}}^{\alpha} \varphi(t)=\varphi(t)+c_{0}+c_{1} t+c_{2} t^{2}+\ldots+c_{n-1} t^{n-1},
$$

for arbitrary $c_{i} \in \mathbf{R}, i=0, \ldots, n$ and $n=[\alpha]+1$.

The following Banach space plays a fundamental role in our analysis. We let $C([0,1], \mathbf{R})$ denote the space of all continuous functions defined on $[0,1]$. The space

$$
E=\left\{u: y \in C([0,1], \mathbf{R}),{ }^{c} D_{0^{+}}^{\sigma} y \in C([0,1], \mathbf{R}), 0<\sigma<1\right\},
$$

endowed with the norm

$$
\|u\|_{E}=\max _{t \in[0,1]}|u|_{E}+\max _{t \in[0,1]}\left|{ }^{c} D_{0^{+}}^{\sigma} u\right|_{E},
$$

is a Banach space.

Lemma 2.12. (Lemma 3.2, [15]) $\left(E,\|.\|_{E}\right)$ is a Banach space.

To study the existence of solution of the problem (1.1)-(1.3), we need the following lemma.

Lemma 2.13. Given $\psi, \eta_{1}, \eta_{2} \in C([0,1], \mathbf{R})$ and $1<q \leq 2,0<\sigma<1$. Then the unique solution of the boundary value problem

$$
\left\{\begin{array}{l}
c D_{0^{+}}^{q} u(t)=\psi(t), \quad 0<t<1 \\
u(0)-u^{\prime}(0)=\int_{0}^{1} \eta_{1}(s) u(s) d s \\
u(1)-u^{\prime}(1)=\int_{0}^{1} \eta_{2}(s) u(s) d s
\end{array}\right.
$$

is given by

$$
u(t)=\int_{0}^{1} G(t, s) \psi(s) d s
$$

where 
$G(t, s)=\left\{\begin{array}{l}\frac{(t-s)^{q-1}}{\Gamma(q)}+\frac{\left(k_{1}-k_{2}-k_{3} t\right)}{\Gamma(q)} \int_{s}^{1} \eta_{1}(r)(r-s)^{q-1} d r \\ +\frac{\left(k_{4}+k_{5} t\right)}{\Gamma(q)} \int_{s}^{1} \eta_{2}(r)(r-s)^{q-1} d r \\ -\frac{\left(k_{4}+k_{5} t\right)}{\Gamma(q-1)}(1-s)^{\alpha-2}-\frac{\left(k_{4}+k_{5} t\right)}{\Gamma(q)}(1-s)^{q-1}, \quad 0 \leq s \leq t \leq 1 . \\ \frac{\left(k_{1}-k_{2}-k_{3} t\right)}{\Gamma(q)} \int_{s}^{1} \eta_{1}(r)(r-s)^{q-1} d r+\left(k_{4}+k_{5} t\right) \int_{s}^{1} \eta_{2}(r)(r-s)^{q-1} d r \\ -\frac{\left(k_{4}+k_{5} t\right)}{\Gamma(q-1)}(1-s)^{q-2}-\frac{\left(k_{4}+k_{5} t\right)}{\Gamma(q)}(1-s)^{q-1}, \quad 0 \leq t \leq s \leq 1,\end{array}\right.$

and $G(t, s)$ is called the Green's function of the boundary value problem (1.1)-(1.3).

Here

$k_{1}=\frac{1}{p_{1}}, \quad k_{2}=\frac{p_{2} p_{3}}{p_{1}^{2} p_{5}}, \quad k_{3}=\frac{p_{3}}{p_{1} p_{5}}$,

$k_{4}=\frac{p_{2}}{p_{1} p_{5}}, \quad k_{5}=\frac{1}{p_{5}}, \quad p_{1}=1-\int_{0}^{1} \eta_{1}(s) d s$,

$p_{2}=1+\int_{0}^{1} s \eta_{1}(s) d s, \quad p_{3}=1-\int_{0}^{1} \eta_{2}(s) d s, \quad p_{4}=2-\int_{0}^{1} s \eta_{2}(s) d s$,

$p_{5}=\left(p_{4}+\frac{p_{2} p_{3}}{p_{1}}\right)$,

where $p_{1}, p_{2}, p_{3}, p_{4}, p_{5}>0$.

Proof. Assume that $u$ is a solution of the boundary value problem (2.4). Then, according to Lemma 2.11, we can reduce the problem (2.4) to an equivalent integral equation

$$
u(t)=c_{0}+c_{1} t+\frac{1}{\Gamma(q)} \int_{0}^{t}(t-s)^{q-1} \psi(s) d s,
$$

for some constants $c_{0}, c_{1} \in \mathbf{R}$.

Taking the fractional integral conditions $u(0)-u^{\prime}(0)=\int_{0}^{1} \eta_{1}(s) u(s) d s$ and $u(1)-u^{\prime}(1)=\int_{0}^{1} \eta_{2}(s) u(s) d s$ into account, it yields

$$
c_{0}-c_{1}=\int_{0}^{1} \eta_{1}(s) u(s) d s .
$$

and

$c_{0}+2 c_{1}+\frac{1}{\Gamma(q)} \int_{0}^{1}(1-s)^{q-1} \psi(s) d s+\frac{1}{\Gamma(q-1)} \int_{0}^{1}(1-s)^{q-2} \psi(s) d s=\int_{0}^{1} \eta_{2}(s) u(s) d s$. 
From (2.7), we have by integration using Fubini's integral theorem and (2.8) and (2.9), we get

$$
\begin{aligned}
c_{1} & =-\frac{p_{3}}{p_{1} p_{5} \Gamma(q)} \int_{0}^{1} \int_{s}^{1} \eta_{1}(r)(r-s)^{q-1} \psi(s) d r d s-\frac{1}{p_{5} \Gamma(q)} \int_{0}^{1}(1-s)^{q-1} \psi(s) d s \\
& -\frac{1}{p_{5} \Gamma(q-1)} \int_{0}^{1}(1-s)^{q-2} \psi(s) d s+\frac{1}{p_{5} \Gamma(q)} \int_{0}^{1} \int_{s}^{1} \eta_{2}(r)(r-s)^{q-1} \psi(s) d r d s .
\end{aligned}
$$

and

$$
\begin{aligned}
c_{0} & =\left(\frac{1}{p_{1} \Gamma(q)}-\frac{p_{2} p_{3}}{p_{1}^{2} p_{5} \Gamma(q)}\right) \int_{0}^{1} \int_{s}^{1} \eta_{1}(r)(r-s)^{q-1} \psi(s) d r d s \\
& +\frac{p_{2}}{p_{1} p_{5} \Gamma(q)} \int_{0}^{1} \int_{s}^{1} \eta_{2}(r)(1-s)^{q-1} \psi(s) d s \\
& -\frac{p_{2}}{p_{1} p_{5} \Gamma(q-1)} \int_{s}^{1}(1-s)^{q-2} \psi(s) d s-\frac{p_{2}}{p_{1} p_{5} \Gamma(q)} \int_{0}^{1}(1-s)^{q-1} \psi(s) d s .
\end{aligned}
$$

Hence the unique solution of problem (2.4) is found by substituting $c_{0}$ and $c_{1}$ by their values in (2.7). This yields

$$
\begin{aligned}
u(t) & =\frac{1}{\Gamma(q)} \int_{0}^{t}(t-s)^{q-1} \psi(s) d s \\
& +\frac{\left(k_{1}-k_{2}-k_{3} t\right)}{\Gamma(q)} \int_{0}^{1} \int_{s}^{1} \eta_{1}(r)(r-s)^{q-1} \psi(s) d r d s \\
& +\frac{\left(k_{4}+k_{5} t\right)}{\Gamma(q)} \int_{0}^{1} \int_{s}^{1} \eta_{2}(r)(r-s)^{q-1} \psi(s) d r d s \\
& -\frac{\left(k_{4}+k_{5} t\right)}{\Gamma(q-1)} \int_{0}^{1}(1-s)^{q-2} \psi(s) d s \\
& -\frac{\left(k_{4}+k_{5} t\right)}{\Gamma(q)} \int_{0}^{1}(1-s)^{q-1} \psi(s) d s \\
& =\int_{0}^{1} G(t, s) \psi(s) d s .
\end{aligned}
$$

This completes the proof.

Lemma 2.14. Assume that $f \in C([0,1) \times \mathbf{R} \times \mathbf{R}, \mathbf{R})$. Then $u \in E$ is a solution of the fractional boundary value problem (1.1)-(1.3) if and only if $\Theta u(t)=u(t)$, for all $t \in[0,1]$.

Proof. Let $u(t) \in E$ be a solution of problem (1.1)-(1.3). Then, using the same method as used in Lemma 2.13, we can obtain that $u$ is a solution of the following integral equation

$$
u(t)=\int_{0}^{1} G(t, s) f\left(s, u(s),{ }^{c} D_{0^{+}}^{\sigma} u(s)\right) d s .
$$

Conversely, let $u \in E$ be a solution of integral equation (2.13). We denote the right-hand side of the first equation in $(2.13)$ by $z(t)$ 


$$
\begin{aligned}
z(t) & =I_{0^{+}}^{q} f\left(s, u(s),{ }^{c} D_{0^{+}}^{\sigma} u(s)\right) \\
& +\frac{\left(k_{1}-k_{2}-k_{3} t\right)}{\Gamma(q)} \int_{0}^{1} \int_{s}^{1} \eta_{1}(r)(r-s)^{q-1} f\left(s, u(s),{ }^{c} D_{0^{+}}^{\sigma} u(s)\right) d r d s \\
& +\frac{\left(k_{4}+k_{5} t\right)}{\Gamma(q)} \int_{0}^{1} \int_{s}^{1} \eta_{2}(r)(r-s)^{q-1} f\left(s, u(s),{ }^{c} D_{0^{+}}^{\sigma} u(s)\right) d r d s \\
& -\frac{\left(k_{4}+k_{5} t\right)}{\Gamma(q-1)} \int_{0}^{1}(1-s)^{q-2} f\left(s, u(s),{ }^{c} D_{0^{+}}^{\sigma} u(s)\right) d s \\
& -\frac{\left(k_{4}+k_{5} t\right)}{\Gamma(q)} \int_{0}^{1}(1-s)^{q-1} f\left(s, u(s),{ }^{c} D_{0^{+}}^{\sigma} u(s)\right) d s .
\end{aligned}
$$

Using Lemma 2.8 and Lemma 2.9, we have

$$
\begin{aligned}
{ }^{c} D_{0^{+}}^{q} z(t) & ={ }^{c} D_{00^{+}}^{q} I_{0^{+}}^{q} f\left(s, u(s),{ }^{c} D_{0^{+}}^{q} u(s)\right) \\
& +\frac{{ }^{c} D_{0^{+}}\left(k_{1}-k_{2}-k_{3} t\right)}{\Gamma(q)} \int_{0}^{1} \int_{s}^{1} \eta_{1}(r)(r-s) q-1 \\
& +\frac{{ }^{c} D_{0^{+}}^{q}\left(k_{4}+k_{5} t\right)}{\Gamma(q)} \int_{0}^{1} \int_{s}^{1} \eta_{2}(r)\left(r-u(s),{ }^{c} D_{0^{+}}^{\sigma} u(s)\right) d r d s \\
& -\frac{{ }^{c} D_{0^{+}}^{q}\left(k_{4}+k_{5} t\right)}{\Gamma-1} f\left(s, u(s),{ }^{c} D_{0^{+}}^{\sigma} u(s)\right) d r d s \\
& -\frac{{ }^{c} D_{0^{+}}\left(k_{4}+k_{5} t\right)}{\Gamma(q)} \int_{0}^{1}(1-s)^{q-2} f\left(s, u(s),{ }^{c} D_{0^{+}}^{\sigma} u(s)\right) d s \\
& =f\left(s, u(s),{ }^{c} D_{0^{+}}^{q} u(s)\right)
\end{aligned}
$$

namely, ${ }^{c} D_{0^{+}}^{q} u(t)=f\left(s, u(s),{ }^{c} D_{0^{+}}^{q} u(s)\right)$. Also it easy to verify that $u$ satisfies conditions (1.2) and (1.3). Hence, $u \in E$ is a solution of the problem (1.1)-(1.3). This achieves the proof.

Let $\Theta: E \rightarrow E$ be the operator defined as

$$
\Theta u(t)=\int_{0}^{1} G(t, s) f\left(s, u(s),{ }^{c} D_{0^{+}}^{\sigma} u(s)\right) d s .
$$

Therefore by Lemma (2.14), the fixed point of operator $\Theta$ coincides with the solution of problem (1.1)-(1.3).

\section{Main results}

In this section, we prove the existence and uniqueness of solution of the boundary vale problem (1.1)-(1.3) in the Banach space E by applying Banach contraction principle [16] and Schauder fixed point theorem [16].

We need the following assumptions to establish our results:

(H1) The function $f:[0,1) \times \mathbf{R} \times \mathbf{R} \rightarrow \mathbf{R}$ is continuous.

(H2) There exists a constant $L>0$ such that: $|f(t, x, \bar{x})-f(t, y, \bar{y})| \leq$ $L(|x-y|+|\bar{x}-\bar{y}|)$, for all $x, \bar{x}, y, \bar{y} \in \mathbf{R}$ and $t \in[0,1]$. 
(H3) There exists a nonnegative function $\beta \in L[0,1]$ such that: $|f(t, x, y)| \leq$ $\beta(t)+\alpha_{1}|x|^{\sigma_{1}}+\alpha_{2}|y|^{\sigma_{2}}$, where $\alpha_{1}, \alpha_{2} \geq 0$ and $0<\sigma_{i}<1$ for $i=1,2$.

(H4) There exists a nonnegative function $\beta \in L[0,1]$ such that:

$|f(t, x, y)| \leq \beta(t)+\alpha_{1}|x|^{\sigma_{1}}+\alpha_{2}|y|^{\sigma_{2}}$,

where $\alpha_{1}, \alpha_{2} \geq 0$ and $\sigma_{1}, \sigma_{2}>1$.

For convenience, we define the following notations:

$$
\begin{aligned}
\omega_{1} & =\frac{1}{q \Gamma(q)}+\frac{k_{1}+k_{2}+k_{3}}{\Gamma(q)} \int_{0}^{1} \int_{s}^{1} \eta_{1}(r)(r-s)^{q-1} d r d s \\
& +\frac{k_{4}+k_{5}}{\Gamma(q)} \int_{0}^{1} \int_{s}^{1} \eta_{2}(r)(r-s)^{q-1} d r d s+\left(k_{4}+k_{5}\right)\left(\frac{1}{\Gamma(q)}+\frac{1}{\Gamma(q+1)}\right), \\
\omega_{2} & =\frac{1}{\Gamma(q)}+\frac{k_{3}}{\Gamma(q)} \int_{0}^{1} \int_{s}^{1} \eta_{1}(r)(r-s)^{q-1} d r d s \\
& +\frac{k_{5}}{\Gamma(q)} \int_{0}^{1} \int_{s}^{1} \eta_{2}(r)(r-s)^{q-1} d r d s \\
& +\frac{k_{5}}{\Gamma(q)}+\frac{k_{5}}{\Gamma(q+1)} \\
\omega_{3} & =\frac{k_{3}}{\Gamma(q)} \int_{0}^{1} \int_{s}^{1} \eta_{1}(r)(r-s)^{q-1} \beta(s) d r d s \\
& +\frac{k_{5}}{\Gamma(q)} \int_{0}^{1} \int_{s}^{1} \eta_{2}(r)(r-s)^{q-1} \beta(s) d r d s \\
& +\frac{k_{5}}{\Gamma(q-1)} \int_{0}^{1}(1-s)^{q-2} \beta(s) d s+\frac{k_{5}}{\Gamma(q)} \int_{0}^{1}(1-s)^{q-1} \beta(s) d s, \\
\omega_{4} & =\max _{0 \leq t \leq 1} \int_{0}^{1}|G(t, s) \beta(s)| d s .
\end{aligned}
$$

Theorem 3.1. Assume that (H1) and (H2) hold. If

$$
L .\left(\omega_{1}+\frac{\omega_{2}}{\Gamma(2-\sigma)}\right)<1 .
$$

Then the boundary value problem (1.1)-(1.3) has a unique solution $u \in E$.

Proof. We prove by using Banach contraction principle [16] and Lemma 2.14 the boundary value problem (1.1)-(1.3) has a unique solution if and only if the operator $\Theta$ has a fixed point in $E$. For this, we need to verify that $\Theta$ is a contraction. Let $u, v \in E$, in view of (2.12) and Lemma (2.14) we get

$$
\Theta u(t)-\Theta v(t)=\int_{0}^{1} G(t, s)\left(f\left(s, u(s),{ }^{c} D_{0^{+}}^{\sigma} u(s)\right)-f\left(s, v(s),{ }^{c} D_{0^{+}}^{\sigma} v(s)\right)\right) d s .
$$


According to (H2), we obtain

$$
\begin{aligned}
|\Theta u(t)-\Theta v(t)| & \leq \int_{0}^{1} G(t, s)\left(f\left(s, u(s),{ }^{c} D_{0^{+}}^{\sigma} u(s)\right)-f\left(s, v(s),{ }^{c} D_{0^{+}}^{\sigma} v(s)\right)\right) d s, \\
& \leq L \int_{0}^{1}|G(t, s)||u(s)-v(s)| d s \\
& +L \int_{0}^{1}|G(t, s)|\left|{ }^{c} D_{0^{+}}^{\sigma} u(s)-{ }^{c} D_{0^{+}}^{\sigma} v(s)\right| d s \\
& \leq L \max _{0 \leq t \leq 1}|u(s)-v(s)| \int_{0}^{1}|G(t, s)| d s \\
& +L \max _{0 \leq t \leq 1}\left|{ }^{c} D_{0^{+}}^{\sigma} u(s)-{ }^{c} D_{0^{+}}^{\sigma} v(s)\right| \int_{0}^{1}|G(t, s)| d s .
\end{aligned}
$$

Indeed, let us estimate the term $\int_{0}^{1}|G(t, s)| d s$, using (2.6) we have

$$
\begin{aligned}
\int_{0}^{1}|G(t, s)| d s & \leq \frac{1}{q \Gamma(q)}+\frac{k_{1}+k_{2}+k_{3}}{\Gamma(q)} \int_{0}^{1} \int_{s}^{1} \eta_{1}(r)(r-s)^{q-1} d r d s \\
& +\frac{k_{4}+k_{5}}{\Gamma(q)} \int_{0}^{1} \int_{s}^{1} \eta_{2}(r)(r-s)^{q-1} d r d s \\
& +\left(k_{4}+k_{5}\right)\left(\frac{1}{\Gamma(q)}+\frac{1}{\Gamma(q+1)}\right) .
\end{aligned}
$$

Consequently (3.2), can be written using the above notation

$$
\begin{aligned}
|\Theta u(t)-\Theta v(t)| & \leq L \omega_{1}\left(\max _{0 \leq t \leq 1}|u(t)-v(t)|+\max _{0 \leq t \leq 1}\left|{ }^{c} D_{0^{+}}^{\sigma} u(t)-{ }^{c} D_{0^{+}}^{\sigma} v(t)\right|\right) \\
& \leq L \omega_{1}\|u(t)-v(t)\| .
\end{aligned}
$$

Since on the other hand we have

$$
{ }^{c} D_{0^{+}}^{\sigma} \Theta u(t)-{ }^{c} D_{0^{+}}^{\sigma} \Theta v(t)=\frac{1}{\Gamma(1-\sigma)} \int_{0}^{t} \frac{(\Theta u)^{\prime}(s)-(\Theta v)^{\prime}(s)}{(t-s)^{\sigma}} d s,
$$

where

$$
(\Theta u)^{\prime}(t)=\int_{0}^{1} \frac{\partial G(t, s)}{\partial t} f\left(s, u(s),{ }^{c} D_{0^{+}}^{\sigma} u(s)\right) d s
$$

and

$\frac{\partial G(t, s)}{\partial t}=\left\{\begin{array}{l}\frac{(t-s)^{q-2}}{\Gamma(q-1)}-\frac{k_{3}}{\Gamma(q)} \int_{s}^{1} \eta_{1}(r)(r-s)^{q-1} d r+\frac{k_{5}}{\Gamma(q)} \int_{s}^{1} \eta_{2}(r)(r-s)^{q-1} d r \\ -\frac{k_{5}}{\Gamma(q-1)}(1-s)^{q-2}-\frac{k_{5}}{\Gamma(q)}(1-s)^{q-1}, \quad 0 \leq s \leq t \leq 1 \\ -\frac{k_{3}}{\Gamma(q)} \int_{s}^{1} \eta_{1}(r)(r-s)^{q-1} d r+\frac{k_{5}}{\Gamma(q)} \int_{s}^{1} \eta_{2}(r)(r-s)^{q-1} d r \\ -\frac{k_{5}}{\Gamma(q-1)}(1-s)^{q-2}-\frac{k_{5}}{\Gamma(q)}(1-s)^{q-1}, \quad 0 \leq t \leq s \leq 1 .\end{array}\right.$

So we get that 


$$
\begin{aligned}
{ }^{c} D_{0^{+}}^{\sigma} \Theta u(t)-{ }^{c} D_{0^{+}}^{\sigma} \Theta v(t) \\
=\frac{1}{\Gamma(1-\sigma)} \int_{0}^{t} \int_{0}^{1}(t-s)^{-\sigma} \frac{\partial G(s, r)}{\partial s}\left(f \left(r, u(r),{ }^{c} D_{0^{+}}^{\sigma} u(r)\right.\right. \\
\left.\quad-f\left(r, v(r),{ }^{c} D_{0^{+}}^{\sigma} v(r)\right)\right) d r d s .
\end{aligned}
$$

Using (H2) yields

$$
\begin{aligned}
& \left|{ }^{c} D_{0^{+}}^{\sigma} \Theta u(t)-{ }^{c} D_{0^{+}}^{\sigma} \Theta v(t)\right| \\
& \leq L \frac{\max _{0 \leq t \leq 1}|u(t)-v(t)|}{\Gamma(1-\sigma)} \int_{0}^{t} \int_{0}^{1}(t-s)^{-\sigma}\left|\frac{\partial G(s, r)}{\partial s}\right| d r d s \\
& +L \frac{\max _{0 \leq t \leq 1}\left|{ }^{c} D_{0^{+}}^{\sigma} u(t)-{ }^{c} D_{0^{+}}^{\sigma} v(t)\right|}{\Gamma(1-\sigma)} \int_{0}^{t} \int_{0}^{1}(t-s)^{-\sigma}\left|\frac{\partial G(s, r)}{\partial s}\right| d r d s .
\end{aligned}
$$

The term $\int_{0}^{1}\left|\frac{\partial G(s, r)}{\partial s}\right| d r$ in (3.6) admits the following estimate

$$
\begin{aligned}
\int_{0}^{1}\left|\frac{\partial G(s, r)}{\partial s}\right| d r & \leq \frac{1}{\Gamma(q)}+\frac{k_{3}}{\Gamma(q)} \int_{0}^{1} \int_{s}^{1} \eta_{1}(r)(r-s)^{q-1} d r d s \\
& +\frac{k_{5}}{\Gamma(q)} \int_{0}^{1} \int_{s}^{1} \eta_{2}(r)(r-s)^{q-1} d r d s \\
& +\frac{k_{5}}{\Gamma(q)}+\frac{k_{5}}{\Gamma(q+1)} .
\end{aligned}
$$

Using the notation given above, (3.6) becomes

$$
\begin{aligned}
\left|{ }^{c} D_{0^{+}}^{\sigma} \Theta u(t)-{ }^{c} D_{0^{+}}^{\sigma} \Theta v(t)\right| & \leq \frac{L \omega_{2}}{\Gamma(2-\sigma)} \max _{0 \leq t \leq 1}|u(t)-v(t)| \\
& +\frac{L \omega_{2}}{\Gamma(2-\sigma)} 0 \leq t \leq 1 \max \left|{ }^{c} D_{0^{+}}^{\sigma} u(t)-{ }^{c} D_{0^{+}}^{\sigma} v(t)\right|, \\
& \leq \frac{L \omega_{2}}{\Gamma(2-\sigma)}\|u(t)-v(t)\|,
\end{aligned}
$$

then

$\|\Theta u(t)-\Theta v(t)\|_{E}=\max _{0 \leq t \leq 1}|\Theta u(t)-\Theta v(t)|+\max _{0 \leq t \leq 1}\left|{ }^{c} D_{0^{+}}^{\sigma} \Theta u(t)-{ }^{c} D_{0^{+}}^{\sigma} \Theta v(t)\right|$.

Thus, it follows from this that

$$
\|\Theta u(t)-\Theta v(t)\| \leq L\left(\omega_{1}+\frac{\omega_{2}}{\Gamma(2-\sigma)}\right)\|u(t)-v(t)\| .
$$

Using the condition (3.1), we conclude that $\Theta$ is a contraction mapping, hence Banach contraction principle guarantees that $\Theta$ has a fixed point which is the unique solution of the boundary value problem (1.1)-(1.3). The proof is complete.

The existence result can be obtained by the well-known Schauder fixed point theorem [16].

Theorem 3.2. Assume that (H1) and (H3) hold. Then the boundary value problem (1.1)-(1.3) has a solution. 
Proof. In view of the continuity of $f$ and $G$, the operator $\Theta$ defined above is continuous.

Firstly, we prove that $\Theta: \mathbf{W} \rightarrow \mathbf{W}$. Let $\mathbf{W}=\left\{u \in E:\|u(t)\|_{E} \leq R, t \in\right.$ $[0,1]\}$ be a bounded subset in $\mathrm{E}$, and choose

$R \geq \max \left\{3 \omega_{5},\left(3 \alpha_{1} \omega_{6}\right)^{\frac{1}{1-\sigma_{1}}},\left(3 \alpha_{2} \omega_{6}\right)^{\frac{1}{1-\sigma_{2}}}\right\}$. For $u \in \mathbf{W}$, and using (H3), we obtain

$$
\begin{aligned}
|\Theta u(t)| & \leq \int_{0}^{1}|G(t, s)|\left|f\left(s, u(s),{ }^{c} D_{0^{+}}^{\sigma} u(s)\right)\right| d s \\
& \leq \int_{0}^{1}|G(t, s) \beta(s)| d s+\left(\alpha_{1} R^{\sigma_{1}}+\alpha_{2} R^{\sigma_{2}}\right) \int_{0}^{1}|G(t, s)| d s
\end{aligned}
$$

Using the notation given above, this becomes

$$
|\Theta u(t)| \leq \omega_{4}+\left(\alpha_{1} R^{\sigma_{1}}+\alpha_{2} R^{\sigma_{2}}\right) \omega_{1} .
$$

In addition

$$
\begin{aligned}
\left|(\Theta u(t))^{\prime}\right| & \leq \int_{0}^{1}\left|\frac{\partial G(t, s)}{\partial t}\right|\left|f\left(s, u(s),{ }^{c} D_{0^{+}}^{\sigma} u(s)\right)\right| d s \\
& \leq \int_{0}^{1}\left|\frac{\partial G(t, s)}{\partial t}\right| \beta(s) d s+\left(\alpha_{1} R^{\sigma_{1}}+\alpha_{2}|R|^{\sigma_{2}}\right) \int_{0}^{1}\left|\frac{\partial G(t, s)}{\partial t}\right| d s .
\end{aligned}
$$

The term $\int_{0}^{1}\left|\frac{\partial G(t, s)}{\partial t}\right| \beta(s) d s$ in (3.12) is estimated using (3.5) and Lemma 2.7 with the above notation, leading to

$$
\begin{aligned}
\int_{0}^{1}\left|\frac{\partial G(t, s)}{\partial t}\right| \beta(s) d s & \leq I_{0^{+}}^{q-1} \beta(t)+\frac{k_{3}}{\Gamma(q)} \int_{0}^{1} \int_{s}^{1} \eta_{1}(r)(r-s)^{q-1} \beta(s) d r d s \\
& +\frac{k_{5}}{\Gamma(q)} \int_{0}^{1} \int_{s}^{1} \eta_{2}(r)(r-s)^{q-1} \beta(s) d r d s \\
& +\frac{k_{5}}{\Gamma(q-1)} \int_{0}^{1}(1-s)^{q-2} \beta(s) d s \\
& +\frac{k_{5}}{\Gamma(q)} \int_{0}^{1}(1-s)^{q-1} \beta(s) d s, \\
& \leq\left\|I_{0^{+}}^{q-2} \beta\right\|_{L^{1}}+\omega_{3} .
\end{aligned}
$$

Then we obtain

$$
\left|(\Theta u(t))^{\prime}\right| \leq\left\|I_{0^{+}}^{q-2} \beta\right\|_{L^{1}}+\omega_{3}+\left(\alpha_{1} R^{\sigma_{1}}+\alpha_{2}|R|^{\sigma_{2}}\right) \omega_{2} .
$$

Consequently, we have 
$\left.\left|{ }^{c} D_{0^{+}}^{\sigma} \Theta u(t)\right| \leq \frac{1}{\Gamma(2-\sigma)}\left(\left\|I_{0^{+}}^{q-2} \beta\right\|_{L^{1}}+\omega_{3}\right)+\frac{1}{\Gamma(2-\sigma)}\left(\alpha_{1} R^{\sigma_{1}}+\alpha_{2} R^{\sigma_{2}}\right) \omega_{2}\right)$.

Then

$$
\|\Theta u(t)\|_{E}=\max _{0 \leq t \leq 1}|\Theta u(t)|+\max _{0 \leq t \leq 1}\left|{ }^{c} D_{0^{+}}^{\sigma} \Theta u(t)\right| .
$$

Therefore, we have

$$
\begin{aligned}
\|\Theta u(t)\|_{E} & \leq \omega_{4}+\frac{1}{\Gamma(2-\sigma)}\left(\left\|I_{0^{+}}^{q-2} \beta\right\|_{L^{1}}+\omega_{3}\right) \\
& +\left(\frac{1}{\Gamma(2-\sigma)} \omega_{2}+\omega_{1}\right)\left(\alpha_{1} R^{\sigma_{1}}+\alpha_{2} R^{\sigma_{2}}\right) \\
& \leq \omega_{5}+\omega_{6}\left(\alpha_{1} R^{\sigma_{1}}+\alpha_{2} R^{\sigma_{2}}\right), \\
& \leq \frac{R}{3}+\frac{R}{3}+\frac{R}{3}=R,
\end{aligned}
$$

where $\omega_{5}=\omega_{4}+\frac{1}{\Gamma(2-\sigma)}\left(\left\|I_{0^{+}}^{q-2} \beta\right\|_{L^{1}}+\omega_{3}\right)$ and $\omega_{6}=\frac{1}{\Gamma(2-\sigma)} \omega_{2}+\omega_{1}$. Therefore we conclude that $\|\Theta u(t)\|_{E} \leq R$. Since $|\Theta u(t)|$ and $\left|{ }^{c} D_{0^{+}}^{\sigma} \Theta u(t)\right|$ are continuous on $[0,1]$, hence $\Theta: \mathbf{W} \rightarrow \mathbf{W}$.

Now, we show that $\Theta$ is completely continuous operator. In fact, let $M=\max _{0 \leq t \leq 1}\left|f\left(t, u(t),{ }^{c} D_{0^{+}}^{\sigma} u(t)\right)\right|$ where $0 \leq t \leq 1$ and $\|u\| \leq R$, then for all $t_{1}, t_{2} \in[0,1], t_{1}<t_{2}$ and $u \in \mathbf{W}$, we have

$$
\begin{aligned}
\left|\Theta u\left(t_{1}\right)-\Theta u\left(t_{2}\right)\right| & =\int_{0}^{1}\left|G\left(t_{1}, s\right)-G\left(t_{2}, s\right)\right|\left|f\left(t, u(t),{ }^{c} D_{0^{+}}^{\sigma} u(t)\right)\right| d s \\
& \leq M \int_{0}^{1}\left|G\left(t_{1}, s\right)-G\left(t_{2}, s\right)\right| d s, \\
& \leq \frac{M\left|t_{1}^{q}-t_{2}^{q}\right|}{\Gamma(q+1)}+\frac{M k_{3}\left|t_{1}-t_{2}\right|}{\Gamma(q)} \int_{0}^{1} \int_{s}^{1} \eta_{1}(r)(r-s)^{q-1} d r d s \\
& +\frac{M k_{5}\left|t_{1}-t_{2}\right|}{\Gamma(q)} \int_{0}^{1} \int_{s}^{1} \eta_{2}(r)(r-s)^{q-1} d r d s \\
& +\frac{M k_{5}\left|t_{1}-t_{2}\right|(q+1)}{\Gamma(q+1)}
\end{aligned}
$$

In addition

$$
\begin{aligned}
& \left|{ }^{c} D_{0^{+}}^{\sigma} \Theta u\left(t_{1}\right)-{ }^{c} D_{0^{+}}^{\sigma} \Theta u\left(t_{2}\right)\right| \\
& =\left|\frac{1}{\Gamma(1-\sigma)}\left(\int_{0}^{t_{1}}\left(t_{1}-s\right)^{-\sigma}(\Theta u(s))^{\prime} d s-\int_{0}^{t_{2}}\left(t_{2}-s\right)^{-\sigma}(\Theta u(s))^{\prime} d s\right)\right|, \\
& \leq \frac{1}{\Gamma(1-\sigma)}\left(\int_{0}^{t_{1}}\left(\left(t_{1}-s\right)^{-\sigma}-\left(t_{2}-s\right)^{-\sigma}\right)\left|(\Theta u(s))^{\prime}\right| d s\right) \\
& +\frac{1}{\Gamma(1-\sigma)} \int_{t_{1}}^{t_{2}}\left(t_{2}-s\right)^{-\sigma}\left|(\Theta u(s))^{\prime}\right| d s
\end{aligned}
$$


From (3.14), we obtain

$$
\begin{aligned}
& \left|{ }^{c} D_{0^{+}}^{\sigma} \Theta u\left(t_{1}\right)-{ }^{c} D_{0^{+}}^{\sigma} \Theta u\left(t_{2}\right)\right| \\
& \leq \frac{\left\|I_{0^{+}}^{q-2} \beta\right\|_{L^{1}}+\omega_{3}+\left(\alpha_{1} R^{\sigma_{1}}+\alpha_{2}|R|^{\sigma_{2}}\right) \omega_{2}}{\Gamma(1-\sigma)}\left(\int_{0}^{t_{1}}\left(\left(t_{1}-s\right)^{-\sigma}-\left(t_{2}-s\right)^{-\sigma}\right) d s\right), \\
& \leq \frac{\left\|I_{0^{+}}^{q-2} \beta\right\|_{L^{1}}+\omega_{3}+\left(\alpha_{1} R^{\sigma_{1}}+\alpha_{2}|R|^{\sigma}\right) \omega_{2}}{\Gamma(2-\sigma)}\left(\left(t_{1}^{1-\sigma}-t_{2}^{1-\sigma}\right)+2\left(t_{2}-t_{1}\right)^{1-\sigma}\right),
\end{aligned}
$$

Since the functions $t_{1}^{q}-t_{2}^{q}, t_{1}-t_{2}, t_{1}^{1-\sigma}-t_{2}^{1-\sigma}$ and $\left(t_{2}-t_{1}\right)^{1-\sigma}$ are uniformly continuous on $[0,1]$, we conclude that $\Theta \mathbf{W}$ is equicontinuous. Also, $\Theta \mathbf{W}$ is uniformly bounded set. So $\Theta \mathbf{W} \subset \mathbf{W}$. By the Arzela-Ascoli Theorem [16], $\Theta: \mathbf{W} \rightarrow \mathbf{W}$ is completely continuous. Hence, the Schauder fixed point theorem implies the existence of a solution in $\mathbf{W}$ for the boundary value problem (1.1)-(1.3). The proof is complete.

Theorem 3.3. Assume that (H1) and (H4) hold. Then the nonlinear boundary value problem (1.1)-(1.3) has a solution.

Proof. The proof is similar to that of Theorem 3.2, so it is omitted.

\section{Examples}

OIn this section, two examples are given to demonstrate the applicability of the above results.

\subsection{Example}

Consider the following boundary value problem

$$
\begin{aligned}
{ }^{c} D_{0^{+}}^{\frac{3}{2}} u(t)= & \frac{2+u(t)+{ }^{c} D_{0^{+}}^{\frac{1}{2}} u(t)}{(13 \sqrt{\pi}+1)\left(3+u(t)+{ }^{c} D_{0^{+}}^{\frac{1}{2}} u(t)\right)}, \quad 0<t<1, \\
& u(0)-u^{\prime}(0)=\int_{0}^{1} s u(s) d s \\
& u(1)-u^{\prime}(1)=\int_{0}^{1} s^{2} u(s) d s .
\end{aligned}
$$


We have

$$
f\left(t, u(t),{ }^{c} D_{0^{+}}^{\frac{1}{2}} u(t)\right)=\frac{2+u(t)+{ }^{c} D_{0^{+}}^{\frac{1}{2}} u(t)}{(13 \sqrt{\pi}+1)\left(3+u(t)+{ }^{c} D_{0^{+}}^{\frac{1}{2}} u(t)\right)} .
$$

Here $q=\frac{3}{2}, \sigma=\frac{1}{2}, \eta_{1}(s)=s, \eta_{2}(s)=s^{2}$, then we can compute $k_{1}, k_{2}, k_{3}, k_{4}, k_{5}, p_{1}, p_{2}, p_{3}, p_{4}, p_{5}$ :

$$
\begin{array}{lll}
p_{1}=1-\int_{0}^{1} s d s=0.5>0, & p_{2}=1+\int_{0}^{1} s^{2} d s=1.3333333>0, \\
p_{3}=1-\int_{0}^{1} s^{2} d s=0.6666666>0, & p_{4}=2-\int_{0}^{1} s^{3}(s) d s=1.75>0, \\
p_{5}=p_{4}+\frac{p_{2} p_{3}}{p_{1}}=3.5277777>0, & k_{1}=\frac{1}{p_{1}}=2>0, \\
k_{2}=\frac{p_{2} p_{3}}{p_{1}^{2} p_{5}}=1.0078738>0, & k_{3}=\frac{p_{3}}{p_{1} p_{5}}=0.3779527>0, \\
k_{4}=\frac{p_{2}}{p_{1} p_{5}}=0.7590553>0, & k_{5}=\frac{1}{p_{5}}=0.2834645>0 .
\end{array}
$$

Set

$$
f(t, x, y)=\frac{2+x+y}{(13 \sqrt{\pi}+1)(3+x+y)} \quad(t, x, y) \in[0,1] \times \mathbf{R} \times \mathbf{R} .
$$

It is clear that $f$ is continuous.

For all $x, \bar{x}, y, \bar{y} \in \mathbf{R}$ and $t \in[0,1]$, we have

$$
\begin{aligned}
f(t, x, y)-f(t, \bar{x}, \bar{y}) & =\frac{1}{(13 \sqrt{\pi}+1)}\left|\frac{2+x+y}{3+x+y}-\frac{2+\bar{x}+\bar{y}}{3+\bar{x}+\bar{y}}\right|, \\
& \leq \frac{1}{(13 \sqrt{\pi}+1)}\left|\frac{x-\bar{x}+y-\bar{y}}{(3+x+y)(3+\bar{x}+\bar{y})}\right|, \\
& \leq \frac{1}{(13 \sqrt{\pi}+1)}(x-\bar{x}+y-\bar{y}), \\
& \leq \frac{1}{(13 \sqrt{\pi}+1)}(x-\bar{x}+y-\bar{y}) .
\end{aligned}
$$

Then the assumption (H2) holds with $L=\frac{1}{(13 \sqrt{\pi}+1)}=0.0415940$

In addition, let us estimate the term $\left(\omega_{1}+\frac{\omega_{2}}{\Gamma(2-\sigma)}\right)$. We have

$$
\begin{aligned}
& \left(\omega_{1}+\frac{\omega_{2}}{\Gamma(2-\sigma)}\right)=\frac{1}{\Gamma(2-\sigma) \Gamma(q)} \\
& +\left(\frac{k_{1}+k_{2}+k_{3}}{\Gamma(q)}+\frac{k_{3}}{\Gamma(2-\sigma) \Gamma(q)}\right) \int_{0}^{1} \int_{s}^{1} \eta_{1}(r)(r-s)^{q-1} d r d s \\
& +\left(\frac{k_{4}+k_{5}}{\Gamma(q)}+\frac{k_{5}}{\Gamma(2-\sigma) \Gamma(q)}\right) \int_{0}^{1} \int_{s}^{1} \eta_{2}(r)(r-s)^{q-1} d r d s+\frac{\left(k_{4}+k_{5}\right)(q+1)}{\Gamma(q+1)} \\
& +\frac{k_{5}(q+1)}{\Gamma(2-\sigma) \Gamma(q+1)}, \\
& =\frac{1}{\Gamma(2-\sigma) \Gamma(q)} \\
& +\left(\frac{k_{1}+k_{2}+k_{3}}{\Gamma(q)}+\frac{k_{3}}{\Gamma(2-\sigma) \Gamma(q)}\right) \int_{0}^{1} \int_{0}^{s} \eta_{1}(s)(s-r)^{q-1} d r d s \\
& +\left(\frac{k_{4}+k_{5}}{\Gamma(q)}+\frac{k_{5}}{\Gamma(2-\sigma) \Gamma(q)}\right) \int_{0}^{1} \int_{0}^{s} \eta_{2}(s)(s-r)^{q-1} d r d s+\frac{\left(k_{4}+k_{5}\right)(q+1)}{\Gamma(q+1)} \\
& +\frac{k_{5}(q+1)}{\Gamma(2-\sigma) \Gamma(q+1)} .
\end{aligned}
$$

By substituting values for $k_{1}, k_{2}, k_{3}, k_{4}, k_{5}, p_{1}, p_{2}, p_{3}, p_{4}, p_{5}, q, \sigma, \eta_{1}$ and $\eta_{2}$, we obtain 
$\frac{1}{\Gamma(2-\sigma)}=\frac{1}{\Gamma\left(2-\frac{1}{2}\right)}=\frac{2}{\sqrt{\pi}}=1.1283791, \frac{1}{\Gamma(q)}=\frac{1}{\Gamma\left(\frac{3}{2}\right)}=1.1283791, \frac{1}{\Gamma(q+1)}$

$=\frac{1}{\Gamma\left(\frac{5}{2}\right)}=\frac{4}{3 \sqrt{\pi}}=0.7522526$, with $\int_{0}^{1} \int_{0}^{s} \eta_{1}(s)(s-r)^{q-1} d r d s=\int_{0}^{1} \int_{0}^{s} s(s-$ $r)^{q-1} d r d s=0.1904761$, and $\int_{0}^{1} \int_{0}^{s} \eta_{1}(s)(s-r)^{q-1} d r d s$

$=\int_{0}^{1} \int_{0}^{s} s^{2}(s-r)^{q-1} d r d s=0.1481481$, in (4.4) we get the estimated term as: $\left(\omega_{1}+\frac{\omega_{2}}{\Gamma(2-\sigma)}\right)=5.6346356$. By computations, we can obtain

$L\left(\omega_{1}+\frac{\omega_{2}}{\Gamma(2-\sigma)}\right)=0.2343670<1$. Hence condition (3.1) is satisfied. Thus Theorem ?? guarantees the uniqueness of a solution for the boundary (4.1)-(4.3) defined on $[0,1]$.

\subsection{Example}

Consider the following boundary value problem

$$
\begin{gathered}
{ }^{c} D_{0^{+}}^{\frac{3}{2}} u(t)=\frac{\left(2 t-\frac{3}{4}\right)^{23 t}}{2+t^{4}}+\frac{\sin \pi t}{2 \sqrt{\pi+1}} u(t)^{\sigma_{1}}+\frac{3^{-2 t}}{7+{ }^{c} D_{0^{+}}^{\frac{1}{2}} u(t)}{ }^{c} D_{0^{+}}^{\frac{1}{2}} u(t)^{\sigma_{2}}, \\
0<t<1 \\
u(0)-u^{\prime}(0)=\int_{0}^{1} s u(s) d s \\
u(1)-u^{\prime}(1)=\int_{0}^{1} s^{2} u(s) d s .
\end{gathered}
$$

We have

$$
\begin{aligned}
f\left(t, u(t),{ }^{c} D_{0^{+}}^{\frac{1}{2}} u(t)\right) & =\frac{\left(2 t-\frac{3}{4}\right)^{23 t}}{2+t^{4}}+\frac{\sin \pi t}{2 \sqrt{\pi+1}} u(t)^{\sigma_{1}} \\
& +\frac{3^{-2 t}}{7+{ }^{c} D_{0^{+}}^{\frac{1}{2}} u(t)}{ }^{c} D_{0^{+}}^{\frac{1}{2}} u(t)^{\sigma_{2}} .
\end{aligned}
$$

It is clear that $f$ is continuous.

Here $q=\frac{3}{2}, \sigma=\frac{1}{2} \eta_{1}(s)=s, \eta_{2}(s)=s^{2}$. Using the same calculations to get the same values, in accordance with the previously mentioned example $p_{1}=0.5>0, p_{2}=1.3333333>0, p_{3}=0.6666666>0, p_{4}=1.75, p_{5}=$ $3.5277777>0, k_{1}=2, k_{2}=1.0078738>0, k_{3}=0.3779527>0, k_{4}=$ $0.7590553>0, k_{5}=0.2834645>0$.

Therefore

$$
f\left(t, u(t),{ }^{c} D_{0^{+}}^{\frac{1}{2}} u(t)\right)<\beta(t)+\left.\alpha_{1} x(t)\right|^{\sigma_{1}}+\alpha_{2} y(t)^{\sigma_{2}},
$$

where $\beta(t)=\frac{\left(2 t-\frac{3}{4}\right)^{23 t}}{2+t^{4}}, \alpha_{1}=\frac{1}{2 \sqrt{\pi}}, \alpha_{2}=\frac{3}{7}$. For $0<\sigma_{1}, \sigma_{2}<1$, the assumption (H3) holds, and for $\sigma_{1}, \sigma_{2}>1$ the assumption (H4) holds. 
Hence by Theorem 3.2 and Theorem 3.3, the boundary value problem (4.5)(4.7) has a solution defined on $[0,1]$.

\section{Acknowledgements}

The author is grateful to the reviewers for carefully reading the paper and for their valuable comments and suggestions. This research work is supported by the General Direction of Scientific Research and Technological Development (DGRSDT), Algeria.

\section{References}

[1] B. Ahmad and S. K. Ntouyas, "Existence results for a coupled system of Caputo type sequential fractional differential equations with nonlocal integral boundary conditions", Applied mathematics and computation, vol. 266, no. 1, pp. 615-622, 2015, doi: 10.1016/j.amc.2015.05.116

[2] R. L. Bagley and P. L. Torvik, "A theoretical basis for the application of fractional calculus to viscoelasticity", Journal of Rheology, vol. 27, no. 3, pp. 201-210, 1983, doi: 10.1122/ 1.549724

[3] M. Benchohra, J. R. Graef and S. Hamani, "Existence results for boundary value problems with non-linear fractional differential equations", Applicable Analysis, vol. 87, no. 7, pp. 851-863, 2008, doi:10.1080/ 00036810802307579

[4] F. Chen, J. J. Nieto and Y. Zhou, "Global attractivity for non-linear fractional differential equations", Nonlinear Analysis Real World Applications, vol. 13, no. 1, pp. 287-298, 2012, doi: 10.1016/j.nonrwa.2011.07.034

[5] D. Delbosco and L. Rodino, "Existence and uniqueness for a nonlinear fractional differential equation", Journal of Mathematical Analysis and Applications, vol. 204, no. 2, pp. 609-625, 1996, doi:10.1006/jmaa.1996.0456 
[6] A. Guezane-Lakoud and R. Khaldi, "Solvability of a fractional boundary value problem with fractional integral condition", Nonlinear Analysis: Theory, Methods and Applications, vol 75, no. 4, pp. 2692-2700, 2012, doi:10.1016/j.na.2011.11.014

[7] R. Hilfer, Applications of Fractional Calculus in Physics. Singapore: World Scientific, 2000.

[8] J. Henderson, R. Luca and A. Tudorache, "On a system of fractional differential equations with coupled integral boundary conditions", Fractional Calculus and Applied Analysis, vol. 18, no. 2, pp. 361-386, 2015, doi:10.1515/ fca-2015-0024

[9] A. A. Kilbas, H. M. Srivastava and J.J. Trujillo, Theory and Applications of Fractional Differential Equations. Amsterdam: Elsevier B.V, 2006.

[10] V. Lakshmikantham and A. S. Vatsala, "Basic theory of fractional differential equations", Nonlinear Analysis: Theory, Methods and Applications, vol. 69, no. 8, pp. 2677-2681, 2008, doi: 10.1016/j.na.2007.08.042

[11] C. Li and W. Deng, "Remarks on fractional derivatives", Applied Mathematics and Computation, vol. 187, no. 2, pp. 777-784, 2007, doi:10.1016/j.amc.2006.08.163

[12] F. Meral, T. Royston and R. Magin, "Fractional calculus in viscoelasticity: an experimental study", Communications in Nonlinear Science and Numerical Simulation, vol. 15, no. 4, pp. 939-945, 2010, doi:10.1016/j.cnsns.2009.05.004

[13] I. Podlubny, Fractional Differential Equations, Mathematics in Science and Engineering. New York: Academic Press, 1999.

[14] M. Rehman and R. Khan, "Existence and uniqueness of solutions for multi-point boundary value problems for fractional differential equations", Applied Mathematics Letters, vol. 23, no. 9, pp. 1038-1044, 2010, doi:10.1016/j.aml.2010.04.033

[15] X. Su, "Boundary value problem for a coupled system of nonlinear fractional differential equations", Applied Mathematics Letters, vol. 22, no. 1, pp. 64-69, 2009, doi:10.1016/j.aml.2008.03.001 
[16] Y. Zhou, Basic Theory of Fractional Differential Equations. Singapore: World Scientific, 2014.

\author{
Ahcene Boukehila \\ Laboratory of Pure and Applied Mathematics, \\ University Amar Telidji of Laghouat, \\ P. O. BOX 37G, Laghouat 03000, \\ Algeria \\ e-mail: a.boukehila@lagh-univ.dz
}

